expressed in the thymus or proteins as high mobility group box proteins 1 and 2 targeted which are sensors of nucleic acidmediated innate immune responses. In addition, the authors used sera from TLR 7 or 9, TLR7 and TLR9-deficient lupusprone MRL-lpr mice to analyse the Toll 7/9 dependence of the autoantigens identified. RF, hnRNPs (A1, A2/B1, A3, D, AB) and anti-chromatin autoantibodies are TLR 7 and 9-dependent and influenced by Tir8, whereas other RA autoantigens such as anti-tissue transglutaminase 2, vimentin and RA-specific anticitrulline antibodies are independent of Toll 7/9 and Tir8.

Conclusion Up to $90 \%$ of all autoantibodies generated are dependent on the MyoD88 innate immune system. Autoantibodies are still generated to proteins that may escape negative selection or are additional sensors of nucleic acidmediated innate immune responses. Autoantibodies to nonRNA or DNA-containing proteins and citrullinated proteins are TLR $7 / 9$ independent and anti-citrulline-specific B cells appear to be dependent on help from $\mathrm{T}$ cells.

\title{
REFERENCE
}

1. Herlands RA, Christensen SR, Sweet RA, et al. Immunity 2008;29:249-60.

\section{A97 TOLL-LIKE RECEPTOR (TLR-7/9), MYOD88, TIR8, DEPENDENT AND INDEPENDENT AUTOANTIGENS}

B Marklein, 1 Z Konthur, ${ }^{2}$ T Häupl, ${ }^{1}$ A Cope, ${ }^{3}$ M Shlomchik, ${ }^{4}$ G Steiner, ${ }^{5}$ G Burmester, ${ }^{1} \mathrm{~K}$ Skriner ${ }^{1}{ }^{1}$ Department of Rheumatology and Clinical Immunology, Humboldt University and Free University, Berlin, Germany; ${ }^{2}$ Department of Vertebrate Genomics, Max Planck Institute for Molecular Genetics, Berlin, Germany; ${ }^{3}$ Department of Rheumatology, King's College, London, UK; ${ }^{4}$ Yale School of Medicine, New Haven, Connecticut, USA; ${ }^{5}$ Division of Rheumatology, Internal Medicine III, Vienna Medical University, Vienna, Austria

\subsection{6/ard.2010.129627j}

Objective This study was conducted with sera from patients with rheumatoid arthritis (RA), systemic lupus erythematosus (SLE) and arthritis and lupus-like disease animal models to identify innate immune system-dependent and -independent autoantigens.

Methods Using protein filter technology (28000 human protein filter, RZPD, Berlin, Germany), the autoantigen profile (autoantigen fingerprint) of RA and SLE patients, mouse collagen and zymosan-induced arthritis, as well as collagen and pristan-induced arthritis in rats and TLR7, TLR9-deficient double-deficient and MyoD88 and Tir8-deficient mice MRLlpr/lpr background were obtained. ${ }^{1}$

Results By high throughput methods within protein filters, over 200 clones were identified to bind similar patient, mouse and rat immunoglobulins. More than half of all autoantigenic proteins in mouse, rat and humans were nucleic acid binding proteins. The authors found that up to $90 \%$ of all autoantigens, for example anti-histone, snRNP, hnRNP, HSP, were MyoD88-dependent and influenced by Tir8. They found 18 identical proteins targeted in human and animal arthritis. These data identified mRNA binding hnRNPs (A1, A2/B1, A3, $\mathrm{D}, \mathrm{A} / \mathrm{B})$ proteins which are part of $\mathrm{P}$ bodies, stress granules and components of messenger RNA stability complex as well as CRP binding proteins as target molecules in mice, rats and humans with RA. In contrast, autoantigens to citrullinated protein enzymes and modified enzymes are generated independently of MyoD88 Toll7, 9 and Tir8. Moreover, the authors found MyoD88-independent autoantigens which are not 